INTERNATIONAL JOURNAL OF RESEARCHES IN BIOSCIENCES, AGRICULTURE AND TECHNOLOGY (C) VISHWASHANTI MULTIPURPOSE SOCIETY (Global Peace Multipurpose Society) R. No. MH-659/13(N) www.vmsindia.org

\title{
SKIN CARE AND CURE WITH PHOTOTHERAPY LAMPS BELONGING TO XENON HALIDE FAMILY
}

\author{
A. B. Lad and U. R. Kathale \\ Amolakchand Mahavidyalaya,Yavatmal \\ drajay_lad@rediffmail.com
}

\begin{abstract}
:
With advance research over last few decades skin diseases like vitiligo,psoriasis, baby jaundice etc. are treated with phototherapy. The stable vitiligo can be treated with the excimer lamps based on excitation of molecule like XeCl. It is used in producing UVB with $308 \mathrm{~nm}$ wavelength which has high penetrating power and is found to be useful in accelerating melanin formation activity. The depigmented skin showing white patch can be repigmented to achieve normal skin colour. The UVB units are available in various shapes and sizes to cover entire body surface or targetting to a small skin area like fingers or toes. Skin care with light sources is in practice since quite long. Systematic study of lamps having different wavelengths made it possible to bifurcate the wavelengths to be used for various diseases.
\end{abstract}

\section{Introduction:}

Phototherapy came into practice in early years of nineteenth century. Kromayer, a German dermatologist, designed in 1904 a water cooled mercury vapor UV lamp. He was the first to treat vitiligo with artificial UVB. In 1969 Fulton et al. used "black light" UVA tubes for the first time in combination with topical 8-MOP in the treatment of vitiligo. Parrish and Fitzpatrick introduced modern photochemotherapy with 8 -MOP, having a peak sensitivity at $330 \mathrm{~nm}$ and UVA fluorescent tubes. They used fluorescent tubes emitting in the 320 - $380 \mathrm{~nm}$ waveband in the PUVA treatment of vitiligo. Although late effects, e.g. skin carcinogenesis, have rarely been reported in vitiligo, the frequently observed phototoxic responses were considered a severe practical problem. Primarily Mercury lamps were popular though they were not much ecofriendly. With the inert gases lamps focus was shifted to various application areas. In case of Xenon it is found that Xenon Iodide molecule can give radiation of wavelength $253 \mathrm{~nm}$ with photon energy $4.91 \mathrm{eV}$, Xenon Bromide with $282 \mathrm{~nm}$ and $4.41 \mathrm{eV}$, Xenon Chloride with $308 \mathrm{~nm}$ and $4.03 \mathrm{eV}$.The last in the series Xenon Floride with $351 \mathrm{~nm}$ and $3.53 \mathrm{eV}$. Out of these four molecules $\mathrm{XeCl}$ is found to be most suitable for radiation that are useful for the treatment of vitiligo patches.We studied the applications of Xenon-halide molecules in the lamps categorised as Excimers.

\section{Methods:}

In fact phototherapy is based on excitation. The energy radiations are obtained for practical use by an electric discharge. Actually it is used more types of discharge for excimer lamp pumping(some of them are glow discharge, pulsed discharge, capacitive discharge, longitudinal and transverse discharges, volume discharge, spark discharge, microhollow discharge, etc.). Currently, the excimer lamps with capacitive discharge type of excitation namely dielectric barrier discharge [19][20] are the most widely spread. Lamps using this discharge type are already commercially available. In this technology electrodes are not in direct contact with the active medium (plasma) that eliminates any interaction between the discharge and the electrodes, contamination of the active medium with sputtering electrode material, as well as electrode corrosion leading to short operational lifetime of conventional lamp constructions. Moreover, dielectric barrier discharge carries out an effective excitation of gas mixture in wide range of working pressure. Such lamps can be produced in any desired form of radiating surface suitable for any specific tasks.

\section{Mechanism :}

Maximum of an excimer lamp radiation wavelength is specified by a working excimer molecule Excimers are diatomic molecules or complexes of molecules that have stable excited electronic states and an unbound or weakly bound ground state. The operation of excimer lamps is based on the formation of excited dimers (excimers) and the following transition from the bound excited excimer state to a weakly bound ground state resulting to an UV-photon radiation. Originally only homonuclear dimers with a stable excited state but a repulsive ground state were called excimers (excited dimers). The term excimer has since been extended to mean any multi-atom molecule with a thermally unstable (repulsive or only very weakly bonding) ground state. But sometimes one can meet term exciplex meaning anexcited complex. It is also excimer molecule 
but not homonuclear dimer. For example, $\mathrm{Xe}_{2}{ }^{*}$, $\mathrm{Kr}_{2}{ }^{*}, \mathrm{Ar}_{2}{ }^{*}$ are excimer molecules but $\mathrm{XeCl}^{*}$, $\mathrm{KrCl}^{*}, \mathrm{XeBr}^{*}, \mathrm{ArCl}^{*}, \mathrm{Xe}_{2} \mathrm{Cl}^{*}$ are exciplex molecules. The best known include rare gas excimers and rare-gas-halogen excimers. Raregas-halogen trimers, metal excimers, metal-raregas excimers, metal-halogen excimers and raregas-oxygen excimers are also known. [1]

Because of these excimer formations are unstable they disintegrate within a few nanoseconds converting their excitation energy to optical radiation. Because of the excimer molecule nature the difference between their stable excited state and weakly bound ground state amounts from 3.5 to $10 \mathrm{eV}$ that is provided radiation of UV and VUV spectral range. The spectrum of excimer lamp radiation is characterized by an intense narrow emission band. [2] The full-width at half maximum of these bands depends on the kind of working molecule and excitation conditions and amounts from 2 to $15 \mathrm{~nm}$. Hence, the excimer lamps are actually quasimonochromatic light sources. Moreover, about $70-80 \%$ of the whole radiation power of an excimer lamp is concentrated in this one emission band. Such sources are suitable for tasks of spectral-selective irradiation and can even replace lasers in some cases.[3][4][5]

In principle all rare gases and rare-gas halogens can form a slightly bound excited state and thus can form excimers. In most cases the reaction kinetics leading to the formation of a special excimer complex is selective. Thus, it is possible to construct radiation sources with high intensity within certain narrow spectral regions. Most studies of vitiligo treatment with phototherapy set a $75 \%$ repigmentation rate as cosmetically acceptable, and are able to achieve it in 12.5 to $75 \%$ of patients after one year of treatment 17 . By comparison, other studies have found a $43 \%$ improvement with narrow band UVB therapy ${ }^{18}$.

The main benefits of excimer lamps from other sources of UV and VUV radiation are the follows a)high average specific power of UV radiation (up to $1 \mathrm{Watt}$ per cubic centimeter of active medium) b)high energy of emitting photon (form 3.5 to $10 \mathrm{eV}$ ) c)quasimonochromatic radiation with the spectral full-width at half maximum from 2 to $15 \mathrm{~nm} \mathrm{d)} \mathrm{high} \mathrm{power}$ spectral density of UV radiation e) choice of wavelength of $\mathrm{UV}$ radiation maximum for specific purposes f) absence of visible and IR radiation $g$ ) low heating of radiating surface $h$ ) availability of multiple-wavelength UV radiation by the simultaneous excitation of several kind of working excimer molecules I)absence of mercury.

\section{References}

1. Excimerlamp Wikipedia the free encyclopedia

2. Rhodes, Ch.K., ed. (1984). Excimer Lasers. Berlin: Springer. p. 271.

3. B. Gellert, U. Kogelschatz; Kogelschatz (1991). "Generation of Excimer Emission in Dielectric Barrier Discharges". Applied Physics B 52 (1): 14-21.

4. Saburoh Satoh, Takao Tanaka, Satoshi Ihara, Chobei Yamabe (2000). "Stereolithography with $\mathrm{XeCl}$ excimer laser/lamp". In Chen, Xiangli; Fujioka, Tomoo; Matsunawa, Akira. Proceedings of the SPIE. High-Power Lasers in Manufacturing 3888: 264-271.

5. Saburoh Satoh, Takao Tanaka, Satoshi Ihara, Chobei Yamabe (2000). "Excimer lamp stereolithography". In Helvajian, Henry; Sugioka, Koji; Gower, Malcolm C et al. Proceedings of the SPIE. Laser Applications in Microelectronic and Optoelectronic Manufacturing V 3933: 272279.

6. K. Köllner, M.B. Wimmershoff, C. Hintz, M. Landthaler, U. Hohenleutner (2005). "Comparison of the 308-nm excimer laser and a 308-nm excimer lamp with 311-nm narrowband ultraviolet $B$ in the treatment of psoriasis". British Journal of Dermatology 152 (4): 750-754.

7. Reena Rai, CR Shrinivas Phototherapy: An Indian Perceptive Indian J Dermatol 2007: 52(4) 169-75.

8. Mysore V. Targeted phototherapy Indian $\mathrm{J}$ Dermatol Venereol Leprol 2009; 75:119-25

9. Diffey B. L. Ultraviolet phototherapy of skin diseases Physics and Engineering in medicine in the New Millenium IPEM:2000 p. 65-7.

10. Spencer J. M., Hadi SM. The Excimer Laser. J Drugs Dermatol 2004: 3 : 522-5

11. Lapidoth M. Adatto M. David M. Targeted UVB Phototherapy for psoriasis A preliminary study. Clin Exp Dermatol 2007;23:32-4

12. Hadi $\mathbf{S}$ Tinio $\mathbf{P}$ Al-Ghaithi $\mathbf{K}$ et al Treatment of vitiligo using the $308 \mathrm{~nm}$ excimer laser. Photomed laser surg 2006:24:354-7 
13. Balasaraswathi, P., Udaya Kumar, Shrinivas CR UVA and UVB radiations in sunlight Indian J Dermatol Venereol Leprol 2002 :68:198-201

14. Mentener MA, See JA, Amend WJ, Ellis CN et al Proceeding of the psoriasis combination and rotation therapy. $\mathrm{J}$ Am Acad Dermatol 1996; 34: 315-21.

15. Kerscher M, Volkanandt M, Plewig G, Lehmann P. Combination phototherapy of psoriasis with calcipotriol and narrow band UVB. Lancet 1993; 342:923

16. Koo JY, Lowe NJ, Lew-Kaya DA, Vasilopoulos AI, Luc JC, Sefton J, et al. Tazarotene plus UVB therpay in the treatment of psoriasis. J Am Acad Dermatol 2000;43:821-8

17. Nicolaidu E, Antoniou C, Stratigos A, Katsambas AD. Narrowband ultraviolet B
18. phototherapy and 308-nm excimer laser in the treatment of vitiligo: a review. Journal of the American Academy of Dermatology. 2009;60(3):470-477.

19. Scherschun L, Kim J, Lim HW. Narrowband ultraviolet $\mathrm{B}$ is a useful and welltolerated treatment for vitiligo. J Am Acad Dermatol. 2001;44(6):999-1003.

20. U. Konelschatz, B. Eliasson and W. Eg1 (1997). "Dielectric-Barrier Discharges. Principle and Applications". J. Phys Iv France 7 (C4): 4766. doi:10.1051/jp4:1997405.

21. Ulrich Kogelschatz (2003). "DielectricBarrier Discharges: Their History, Discharge Physics, and Industrial Applications". Plasma Chemistry and Plasma Processing 23 (1): 1-46. 